\title{
ORIGINAL
}

\section{INTERRELACION ENTRE CALIDAD, HÁBITOS DE SUEÑO Y AJUSTE ESCOLAR EN ADOLESCENTES DE UN DISTRITO URBANO DE GALICIA}

Teresa J. García-Real (1), Luisa Losada-Puente (1), Iria Vázquez Rodríguez (1) y Tomás M. Díaz-Román (2)

(1) Departamento de Didácticas Específicas y Métodos de Investigación y Diagnóstico en Educación. Facultade de Ciencias da

Educación. Universidade da Coruña. La Coruña. España.

(2) Servicio de Radiodiagnóstico. Hospital Comarcal de Monforte-Lugo. Lugo. España.

Los autores declaran que no existe conflicto de intereses.

\section{RESUMEN}

Fundamentos: La duración y calidad del sueño están relacionadas en los adolescentes con su estado emocional y rendimiento académico. El objetivo del estudio fue evaluar la calidad del sueño en un grupo de jóvenes y explorar su relación con el ajuste y rendimiento escolar.

Métodos: Se realizó un estudio descriptivotransversal, con 56 adolescentes entre 13 y 17 años de un instituto de educación secundaria de un distrito de La Coruña, durante el curso 2016-2017. Los participantes cumplimentaron el Índice de Calidad del Sueño de Pittsburgh (ICSP), la Escala Breve de Ajuste Escolar y un breve cuestionario sobre hábitos de sueño y calificaciones académicas. Realizamos el análisis descriptivo y diferentes pruebas comparativas o de asociación estadística por métodos paramétricos o no paramétricos según cada variable, considerándose significativos valores de $\mathrm{p}<0,10$.

Resultados: El 35,7\% de la muestra se clasificó como mala durmiente y la somnolencia fue la variable más alterada del ICSP. Las mujeres dormían significativamente menos que los varones, aunque ambos retrasaban 100 minutos el sueño durante el fin de semana. Ser buen o mal durmiente no afectó al rendimiento académico. El ajuste escolar fue satisfactorio globalmente. Las variables que expresaron desajuste en la interacción con los profesores y compañeros de clase, así como la realización de tareas escolares, se relacionaron con la disfunción diurna.

Conclusiones: La somnolencia es el problema más relevante en el grupo de malos durmientes. Esta puede influir negativamente en el ajuste escolar y en la motivación para las actividades escolares de todos los adolescentes, siendo frecuente y subestimada en la mayoría de los casos.

Palabras claves: Adolescentes, Calidad del sueño, Hábitos de sueño, Somnolencia, Ajuste escolar, Rendimiento académico.

Correspondencia:

Teresa J. García-Real

Departamento de Didácticas Específicas

y Métodos de Investigación y Diagnóstico en Educación

Facultade Ciencias da Educación

Universidade da Coruña

Campus de Elviña, s/n

15271 La Coruña, España

teresa.greal@udc.es

\section{ABSTRACT \\ Relationship among sleep quality, sleep habits and school adjustment in adolescents from an urban district of Galicia}

Background: The duration and the sleep quality are related to the emotional status and the academic performance in adolescents. The objective of this study was to evaluate the quality of sleep in a group of adolescents, and to explore its relationship with school adjustment and performance.

Methods: A cross-sectional descriptive study was made with 56 adolescents aged 13-17 years from a high school institute in an urban district of La Coruña city was performed during the scholar's year 2016-2017. The participants completed the Pittsburgh Sleep Quality Index (ICSP) and the Brief School Adjustment Scale, and a brief questionnaire about their sleep habits and school scores. Descriptive analysis and different comparative parametric or not parametric statistical tests were applied according to type of variable; statistical significant value was considerate for $\mathrm{p}<0.10$.

Results: The $35.7 \%$ of sample was poor sleepers and sleepiness was the most altered variable in ICSP. The female participants tended to sleep less than the male participants, and all of them went to sleep 100 minutes later on the weekend. Being a good or bad sleeper did not affect academic performance. Globally the school adjustment was satisfactory. Diurnal dysfunction was related to a worse adolescent-teacher and adolescent-classmate relationship. Diurnal dysfunction was also related to a poor motivation to perform academic tasks.

Conclusions: Sleepiness is the most relevant problem in poor sleepers. Sleepiness has a negative influence on school adjustment and motivation for school activities of all adolescents; it is frequent and underestimated in most of the cases.

Key words: Adolescents, Sleep quality, Sleep habits, Sleepiness, School adjustment, School performance.

Cita sugerida: García-Real TJ, Losada-Puente L, Vázquez Rodríguez I, Díaz-Román TM. Interrelacion entre calidad, hábitos de sueño y ajuste escolar en adolescentes de un distrito urbano de Galicia. Rev Esp Salud Pública. 2020; 94: 20 de marzo e202003016 


\section{INTRODUCCIÓN}

El sueño es un regulador de la conducta, la emoción y la atención ${ }^{(1,2)}$. Por ello, la calidad y la duración del sueño no sólo están ligadas al aprendizaje, a la consolidación de la memoria y al rendimiento académico ${ }^{(3,4,5,6)}$, sino que también las alteraciones asociadas a un sueño inadecuado (somnolencia, dificultad en la concentración, apatía e irritabilidad) pueden propiciar problemas en la interacción familiar y social ${ }^{(7)}$. Por este motivo, el sueño es fundamental para mantener la salud y, en particular, para el desarrollo y la maduración cerebral de los adolescentes ${ }^{(8)}$.

Las alteraciones relacionadas con el sueño fluctúan con la edad y con el medio sociocultu$\mathrm{ral}^{(9)}$, si bien las dificultades para iniciar, mantener el sueño sin interrupción o la duración del mismo son frecuentes en los adolescentes ${ }^{(10)}$. En concreto, se han descrito estas alteraciones hasta en un $65 \%$ de los jóvenes entre 16 y 18 años $^{(11)}$. Aunque, entre los mismos adolescentes a diferentes edades muestran hábitos y alteraciones del sueño distintos ${ }^{(12,13)}$. En general, es un hecho comprobado el retraso en las rutinas del sueño durante los fines de semana ${ }^{(14)}$. Así, los adolescentes de 18 años suelen dormir hasta dos horas más los fines de semana respecto a los días escolares, mientras que los jóvenes de 10 a 14 años duermen sólo de 30 a 40 minutos más ${ }^{(15)}$.

Durante la adolescencia, la duración del sueño disminuye conforme aumenta la edad, lo cual parece relacionado con la interacción de factores biológicos, psicológicos y sociales ${ }^{(9)}$. La decisión de irse a dormir forma parte del refuerzo en la autonomía a la que aspira el adolescente ${ }^{(9)}$. Asimismo, existe un mayor uso de los dispositivos tecnológicos conforme aumenta la edad del adolescente, y el tiempo de exposición a la luz proveniente de estos equipos o de otras fuentes afectan al ritmo circadiano, retrasando el sueño ${ }^{(16)}$. No obstante, la necesidad de dormir no disminuye, sino que puede aumentar durante la pubertad ${ }^{(17)}$.
La mala calidad del sueño nocturno puede desencadenar somnolencia diurna ${ }^{(18)}$. Esto puede afectar a la adecuada evolución del adolescente en la escuela, entendida como un paso crucial para el desarrollo de conductas adaptativas en el adolescente ${ }^{(19)}$. La excesiva somnolencia diurna es frecuente entre la población ${ }^{(20,21)}$ y se ha relacionado con el rendimiento académico, la calidad de vida ${ }^{(22,23)}$, los estados de ánimo depresivos, las emociones negativas y un pobre funcionamiento socioemocional ${ }^{(24,25,26)}$.

La relación con los compañeros y los docentes, la integración en grupos, la autoestima, la motivación para asistir a la escuela y las metas académicas son claros indicadores del ajuste escolar ${ }^{(27,28)}$. Junto a este, el rendimiento académico es considerado un elemento clave en el éxito del alumno.

Aunque abundan estudios sobre el rendimiento académico en nuestro país, en general son escasos los que correlacionan el factor sueño con el rendimiento y el ajuste escolar. Destaca un meta-análisis que resume 1.595 trabajos sobre este tema en España ${ }^{(29)}$. En él, se evidenció que el 39,3\% de los estudios analizaban factores personales, familiares o escolares. Sin embargo, dentro de la dimensión personal, sólo el $2 \%$ abordó el factor salud física, y en ninguno de ellos aparecen trabajos sobre el sueño como factor a analizar en el rendimiento académico. Sobre este tema es frecuente encontrar grandes estudios epidemiológicos internacionales que recogen características poblacionales generales y susceptibles de aplicarse en programas de salud. Las pautas derivadas de estos estudios pueden ser complementadas con hallazgos más específicos de contextos socioculturales determinados que satisfagan distintos perfiles de la población.

Es por ello que el objetivo del presente estudio fue evaluar la calidad del sueño y explorar algunos hábitos de sueño en un grupo reducido 
de adolescentes representativos de un entorno urbano, así como explorar posibles relaciones con el ajuste y el rendimiento escolar.

\section{SUJETOS Y MÉTODOS}

La presente investigación desarrolló un estudio descriptivo transversal, limitado a un área urbana de La Coruña. En la propuesta de reorganización por distritos del municipio de La Coruña de $2016^{(30)}$, la población estimada para el distrito 6 era de 23.200 habitantes, lo que representaba el 9,5\% de la población del municipio, estando formada por barrios históricamente asentados y con una identidad común. Dicho distrito contiene dos institutos de enseñanza secundaria, en uno de los cuales se realizó este trabajo durante el curso 2016-2017. Se incluyeron a los alumnos entre 13 y 17 años (media $(\mathrm{M})=14,9$; desviación típica $(\mathrm{DT})=1,06)$, desde $1^{\text {er }}$ curso de ESO hasta $2^{\circ}$ curso de Bachillerato, que quisieron participar de forma voluntaria. Previa inclusión en el estudio, se obtuvo la autorización del centro y de los padres, excluyéndose los alumnos con antecedentes de problemas psiquiátricos diagnosticados. La muestra obtenida se compuso de 56 adolescentes, de los cuales 23 eran varones $(41,1 \%)$ y 33 eran mujeres $(58,9 \%)$.

Instrumentos para evaluar la calidad y hábitos del sueño. Para evaluar la calidad del sueño, se empleó el Índice de Calidad del Sueño de Pittsburgh (ICSP) ${ }^{(31)}$, adaptado al castellano por Macías y Royuela (1996) ${ }^{(32)}$. El ICSP es un cuestionario compuesto por 24 ítems, 19 de los cuales son autocumplimentados por el propio sujeto, mientras que los 5 restantes no son puntuables sino que se usan como control. Las preguntas autocumplimentadas se combinan entre sí para formar siete componentes:

- C1: Calidad subjetiva del sueño.

- C2: Latencia del sueño.
- C3: Duración del sueño.

- C4: Eficiencia del sueño.

- C5: Perturbaciones del sueño.

- C6: Uso de medicación para dormir.

- C7: Disfunción diurna.

En nuestro estudio, este último componente valoró, específicamente, la frecuencia y la severidad de la somnolencia durante las actividades escolares. Cada componente es valorado de 0 (sin dificultad para dormir) a 3 (dificultad grave para dormir). De tal modo que un valor alto de cualquier componente significa siempre peor calidad de sueño. Para obtener la puntuación global se suman los siete componentes, obteniéndose un resultado entre 0 y 21 puntos. Las valoraciones superiores a 5 puntos identifican a los sujetos como malos durmientes.

Se formularon, además, cinco preguntas, las cuales no pertenecían al cuestionario del ICSP. Dos eran de respuesta dicotómica, dirigidas a los padres: "¿Tiene su hijo resistencia a irse a la cama en días escolares?" y "¿Tiene su hijo dificultad para despertar por la mañana?" Otras tres estaban dirigidas al propio alumno: " ¿A qué hora te vas a la cama los días escolares?", "A qué hora te vas a la cama los fines de semana?” y "¿En tu opinión, cuántas horas debería dormir un chico/a de tu edad?"

Instrumentos para valorar el rendimiento académico y el ajuste escolar. Se consultó a los padres acerca de la nota media en matemáticas y en lengua castellana de sus hijos en el último trimestre. Asímismo, para analizar el ajuste escolar se aplicó a los adolescentes la Escala Breve de Ajuste Escolar (EBAE-10) ${ }^{(28)}$.

El EBAE-10 es un instrumento que ofrece un concepto integral del contexto escolar, 
formado por 10 ítems, medidos en escala tipo Likert de 6 puntos (1 significa completamente en desacuerdo y 6 completamente de acuerdo). Los primeros 5 ítems reflejan el ajuste escolar y los 5 últimos el desajuste escolar. En nuestro estudio, se invirtió el sentido de los últimos cinco ítems para que todas las variables pudieran ser interpretadas en sentido creciente como los 5 primeros ítems. El rango de la escala oscila entre 10 y 60 , considerándose mayor ajuste escolar cuanto más elevadas sean las puntuaciones, independientemente del sentido de las preguntas. Todos los ítems pueden verse en la tabla 5 .

Análisis de los datos. Los datos obtenidos fueron analizados con el Paquete estadístico SPSS versión 24. Se llevaron a cabo análisis descriptivos de las variables recogidas. En la comparación entre sexos, dado el mayor número de mujeres, se calcularon porcentajes relativos según el género. Los porcentajes de los componentes del ICSP se compararon a través de las pruebas de Chi cuadrado y del test de Fisher cuando procedió. Se comprobó la normalidad de la distribución de las variables cuantitativas por medio de la prueba de Kolmogorov-Smirnov. Las pruebas comparativas de medias para las notas académicas se realizaron por medio de la prueba T-student. Para el resto de las comparaciones entre medias se empleó la prueba U de Mann Whitney. Se exploró la asociación entre las variables calculando el coeficiente Rho de Spearman. Las diferencias se consideraron estadísticamente significativas para valores de $\mathrm{p}<0,10$.

\section{RESULTADOS}

Calidad del sueño. Tras el análisis del ICSP, la cantidad de malos durmientes detectados fue de un $35,7 \%$ de adolescentes $(\mathrm{n}=20)$, siendo 13 mujeres $(23,2 \%)$ y 7 varones $(12,5 \%)$. Los buenos durmientes estaban representados en un $64,3 \%(n=36)$, de los que 20 eran mujeres $(35,7 \%)$ y 16 varones $(28,6 \%)$.
En la tabla 1 se muestran los porcentajes de las opiniones ofrecidas por los 56 participantes que caracterizaron los siete componentes del ICSP según su gravedad, clasificadas en dos tipos de respuesta: "respuestas favorables" (agrupando las de tipos muy buenas y bastantes buenas, entre 0-1 puntos) y "respuestas desfavorables" (agrupando las muy malas y bastantes malas, entre 2-3 puntos). Los porcentajes de respuestas desfavorables del ICSP se ordenaron de la siguiente manera: disfunción diurna $(55,4 \%)$, latencia del sueño $(26,8 \%)$, calidad subjetiva del sueño $(23,2 \%)$, perturbaciones del sueño $(12,5 \%)$, eficiencia del sueño $(7,1 \%)$, uso de medicación para dormir $(3,6 \%)$ y duración del sueño $(1,8 \%)$.

En todos los componentes del ICSP analizados (figura 1), las mujeres superaron a los varones en "respuestas desfavorables", excepto en los componentes $\mathrm{C} 2(24,2 \%$ frente a $30,4 \%)$ y C6 $(0 \%$ contra $8,7 \%)$. El dato más relevante fue la disminución de "respuestas favorables" en ambos sexos en C7-Disfunción diurna, si bien en ninguno de los casos se alcanzó significación estadística.

Hábitos de sueño. Una vez que se preguntó a los padres sobre la resistencia de sus hijos para irse a la cama en días escolares, el 32,1\% mostraron resistencia (9 mujeres y 9 varones) frente al $67,9 \%$ que no la refirieron ( 24 mujeres y 14 varones). En cuanto al momento de despertarse por las mañanas, los padres del 39,3\% indicaron dificultades en sus hijos (12 mujeres y 10 varones) frente al $60,7 \%$ que dijeron que no tenían problemas (21 mujeres y 13 varones).

Sin embargo, del total de 22 adolescentes con dificultades para despertarse, no todos se encontraban entre los resistentes para ir a dormir. Así, en el caso de los 10 varones con dificultad para despertarse, 6 tenían resistencia para irse a dormir (60\%). En el caso de las 12 mujeres, sólo 3 presentaron resistencia para marcharse a dormir (25\%). 
Tabla 1

Distribución de los adolescentes según las puntuaciones obtenidas en los siete componentes del Índice de Calidad de Sueño de Pittsburgh (ICSP).

\begin{tabular}{|c|c|c|c|c|c|c|c|c|}
\hline \multirow{4}{*}{ Componentes del ICSP } & \multicolumn{8}{|c|}{ Puntuaciones de las respuestas } \\
\hline & \multicolumn{4}{|c|}{ Respuestas favorables } & \multicolumn{4}{|c|}{ Respuestas desfavorables } \\
\hline & \multicolumn{2}{|c|}{ Muy buena } & \multicolumn{2}{|c|}{ Bastante buena } & \multicolumn{2}{|c|}{ Bastante mala } & \multicolumn{2}{|c|}{ Muy mala } \\
\hline & $\mathrm{n}$ & $\%$ & $\mathrm{n}$ & $\%$ & $\mathrm{n}$ & $\%$ & $\mathrm{n}$ & $\%$ \\
\hline C1-Calidad subjetiva del sueño & 24 & 42,9 & 19 & 33,9 & 4 & 7,1 & 9 & 16,1 \\
\hline C2-Latencia del sueño & 26 & 46,4 & 16 & 28,6 & 9 & 16,1 & 5 & 8,9 \\
\hline C3-Duración del sueño & 45 & 80,4 & 10 & 17,9 & 1 & 1,8 & 0 & 0 \\
\hline C4-Eficiencia del sueño & 49 & 87,5 & 3 & 5,4 & 4 & 7,1 & 0 & 0 \\
\hline C5-Perturbaciones del sueño & 3 & 5,4 & 46 & 82,1 & 7 & 12,5 & 0 & 0 \\
\hline C6-Uso de medicación & 52 & 92,9 & 2 & 3,6 & 0 & 0 & 2 & 3,6 \\
\hline C7-Disfunción diurna & 8 & 14,3 & 17 & 30,4 & 21 & 37,5 & 10 & 17,9 \\
\hline
\end{tabular}

\section{Figura 1}

Porcentaje relativo de respuestas favorables/desfavorables en mujeres y varones en cada componente del ICSP.

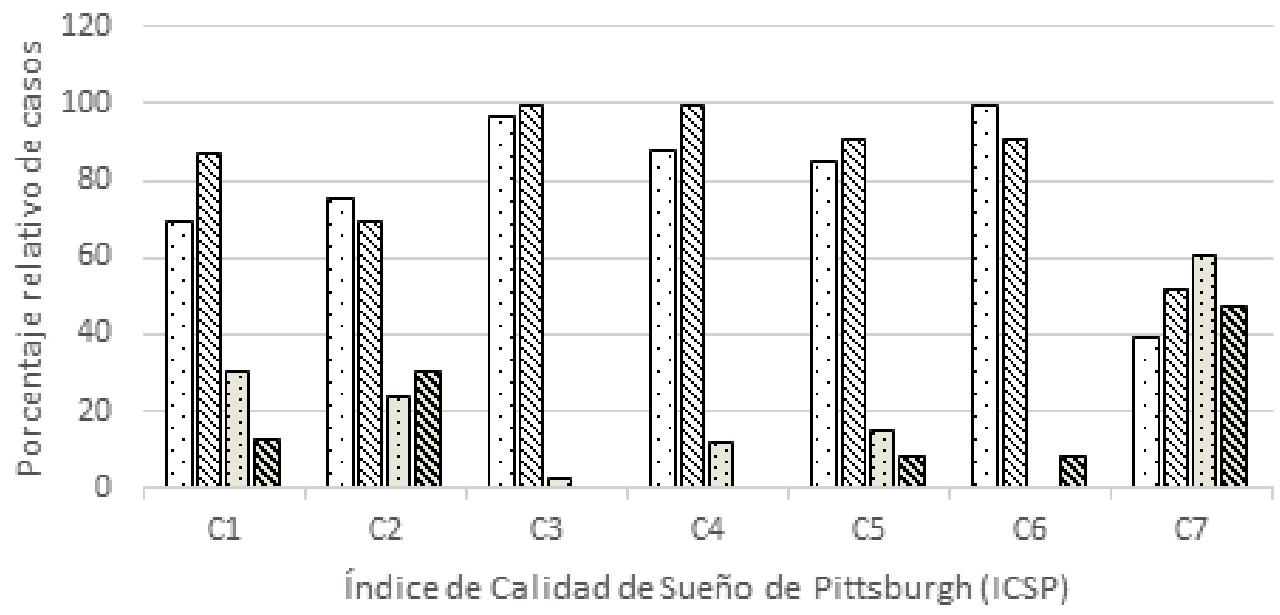

๑Mujer es Resp Favorables $\mathbf{\$}$ Varones Resp Favorables
口Mujer es Resp Desavor ables $\mathbf{\$}$ Varones Resp Desfavorables 
Comparando los valores medios alcanzados en cada componente del ICSP entre los adolescentes con y sin resistencia para irse a dormir, así como en los alumnos con y sin dificultad para despertarse, tal y como se observa en la tabla 2, se encontraron diferencias estadísticamente significativas sólo en el caso del componente C7-Disfunción diurna, que mostró un valor superior en el grupo que tenía dificultades para despertarse $(\mathrm{p}=0,08)$.
La tabla 3 muestra los resultados sobre la hora de irse a la cama en los días escolares y el fin de semana, la duración del sueño y la creencia sobre el tiempo que los alumnos pensaban que deberían dormir. No se hallaron diferencias significativas entre las mujeres y los varones en cuanto a la hora de irse a dormir en días escolares ni en el fin de semana, pero sí en cuanto a la duración del sueño, pues las mujeres dormían 34 minutos menos de media que los varones $(\mathrm{p}=0,04)$.

\section{Tabla 2}

Valores promedios por componente del ICSP entre los subgrupos de adolescentes con resistencia a irse a la cama y dificultades para despertar por la mañana.

\begin{tabular}{|l|c|c|c|c|c|c|c|c|}
\hline \multicolumn{1}{|c|}{ PREGUNTAS } & $\mathrm{C} 1$ & $\mathrm{C} 2$ & $\mathrm{C} 3$ & $\mathrm{C} 4$ & $\mathrm{C} 5$ & $\mathrm{C} 6$ & $\mathrm{C} 7$ & ICSP \\
\hline Sin resistencia a dormir & 0,89 & 1,08 & 0,18 & 0,24 & 1,08 & 0,10 & 1,50 & 5,08 \\
& $(1,01)$ & $(0,88)$ & $(0,46)$ & $(0,59)$ & $(0,43)$ & $(0,51)$ & $(1,01)$ & $(2,80)$ \\
\hline Con resistencia a dormir & 1,11 & 1,05 & 0,28 & 0,11 & 1,06 & 0,22 & 1,78 & 5,61 \\
& $(1,23)$ & $(1,11)$ & $(0,46)$ & $(0,47)$ & $(0,42)$ & $(0,73)$ & $(0,81)$ & $(2,64)$ \\
\hline Sin dificultad para despertar & 1,03 & 1,02 & 0,15 & 0,20 & 1,00 & 0,15 & 1,41 & 4,97 \\
$(1,09)$ & $(0,90)$ & $(0,36)$ & $(0,59)$ & $(0,43)$ & $(0,56)$ & $(0,92)$ & $(2,67)$ \\
\hline Con dificultad para despertar & 0,86 & 1,14 & 0,32 & 0,18 & 1,18 & 0,14 & $\left.1,86^{*}\right)$ & 5,68 \\
& $(1,08)$ & $(1,04)$ & $(0,57)$ & $(0,50)$ & $(1,07)$ & $(0,64)$ & $(0,94)$ & $(2,85)$ \\
\hline
\end{tabular}

Medias (Desviación típica, DT); $(*)$ p $<0,10$. Siglas: C1-Calidad subjetiva del sueño; C2-Latencia del sueño; C3-Duración del sueño; C4-Eficiencia del sueño; C5-Perturbaciones del sueño; C6-Uso de medicación; C7-Disfunción diurna.

\begin{tabular}{|c|c|c|c|c|c|c|}
\hline \multicolumn{7}{|c|}{$\begin{array}{l}\text { Tabla } 3 \\
\text { Valores promedios del horario para dormir, duración del sueño y creencia } \\
\text { sobre el tiempo que deberían dormir entre los distintos sexos. }\end{array}$} \\
\hline \multirow{2}{*}{ Variables } & \multicolumn{2}{|c|}{ Mujeres } & \multicolumn{2}{|c|}{ Varones } & \multicolumn{2}{|c|}{ Total } \\
\hline & M & DT & M & DT & M & DT \\
\hline Hora de ir a dormir días escolares ${ }^{(a)}$ & 23,20 & 0,53 & 23,15 & 1,00 & 23,18 & 0,57 \\
\hline Hora de ir a dormir fin de semana $a^{(a)}$ & 00,53 & 1,07 & 1,05 & 1,23 & 00,58 & 1,13 \\
\hline Duración del sueño ${ }^{(\mathbf{b})}$ & $7,32^{(*)}$ & 0,56 & $8,06^{(*)}$ & 0,56 & 7,45 & 0,58 \\
\hline $\begin{array}{l}\text { Creencia sobre el tiempo que } \\
\text { deberían dormir }{ }^{(b)}\end{array}$ & $8,18^{(*)}$ & 0,32 & $8,47^{(*)}$ & 0,47 & $8,30^{(* *)}$ & 0,41 \\
\hline
\end{tabular}


Ni los varones ni las mujeres cumplieron con el número de horas que pensaban que debían dormir $(\mathrm{p}<0,001)$. En general, ambos sexos dormían como promedio 45 minutos menos respecto a sus expectativas de sueño. Las mujeres pensaron que debían dormir como promedio 29 minutos menos que los varones $(\mathrm{p}=0,02)$. Se observó una correlación negativa entre la duración del sueño y el tiempo que los adolescentes creían que debían dormir $(\mathrm{r}=$ $0,490, \mathrm{p}<0,001)$.

Rendimiento académico y ajuste escolar. Los resultados obtenidos tras comparar las notas de matemáticas y lengua española entre los grupos de buenos y malos durmientes se muestran en la tabla 4. Comparando las notas por sexo y sin tener en cuenta el ICSP, se observó que las mujeres tenían notas significativamente superiores a los varones, tanto en matemáticas como en lengua $(p=0,03)$. Entre los grupos de buenos y malos durmientes no se encontraron diferencias significativas, ni en los valores promedios de la nota de matemáticas ni en los de lengua. Tampoco se demostró correlación significativa entre las notas de matemáticas y lengua con las horas de sueño, la hora de irse a la cama o la hora de despertarse por la mañana.
En la tabla 5 se observan los resultados promedios de las variables del EBAE-10, según los grupos del ICSP y sexo. Comparando las variables del EBAE-10 entre los grupos de la clasificación de ICSP, en la pregunta 1, "Creo que soy buen estudiante", la diferencia entre los buenos y malos durmientes presentó significación estadística $(\mathrm{p}=0,05)$. En la pregunta 6, "Tengo problemas con mis compañeros de clase", los buenos durmientes obtuvieron mejor promedio que los malos durmientes $(\mathrm{p}=0,08)$. Finalmente, en la pregunta 8, "Me siento rechazado por los compañeros", los buenos durmientes tuvieron mejor ajuste escolar que los malos durmientes $(p=0,02)$, y esta diferencia fue más acusada en las mujeres $(p=0,01)$.

Por último, se encontraron varias correlaciones de interés entre las variables de EBAE-10 $\mathrm{y}$ aspectos del sueño evaluados por el ICSP. Todas las mencionadas aquí tuvieron significación estadística con $\mathrm{p}<0,05$ y signo negativo, lo cual significa que valores altos de buen ajuste escolar se asociaron a cifras bajas de ISCP (buenos durmientes). La pregunta 6, "Tengo problemas con mis compañeros", correlacionó con los componentes del ICSP C1-Calidad subjetiva del sueño $(r=-0,319, \quad p=0,016)$,

\begin{tabular}{|c|c|c|c|c|c|c|c|c|}
\hline & Distr & $\begin{array}{l}\text { ción d } \\
\text { y mal }\end{array}$ & $\begin{array}{l}\text { notas } \\
\text { urmier }\end{array}$ & $\begin{array}{l}\text { bla } 4 \\
\text { lémic } \\
\text { del IC }\end{array}$ & $\begin{array}{l}\text { los gr } \\
\text { según }\end{array}$ & $\begin{array}{l}\text { s de b } \\
\text { xo. }\end{array}$ & & \\
\hline & & & & & & & & \\
\hline Variables & Mate & icas & & & Mat & icas & & \\
\hline & M & DT & M & DT & M & DT & M & DT \\
\hline $\begin{array}{l}\text { Buenos } \\
\text { durmientes }\end{array}$ & 6,42 & 2,01 & 7,05 & 1,88 & 4,87 & 2,36 & 5,50 & 1,63 \\
\hline $\begin{array}{l}\text { Malos } \\
\text { durmientes }\end{array}$ & 6,36 & 1,29 & 5,69 & 1,70 & 5,86 & 2,27 & 5,43 & 1,62 \\
\hline $\begin{array}{l}\text { Muestra } \\
\text { total }\end{array}$ & $6,40^{(*)}$ & 1,75 & $6,51^{(*)}$ & 1,90 & $5,17^{(*)}$ & 2,33 & $5,48^{(*)}$ & 1,59 \\
\hline
\end{tabular}




\begin{tabular}{|c|c|c|c|c|c|c|}
\hline \multicolumn{7}{|c|}{$\begin{array}{l}\text { Tabla } 5 \\
\text { Valores promedios del EBAE-10 en los grupos de buenos } \\
\text { y malos durmientes del ICSP según el sexo. }\end{array}$} \\
\hline \multirow{2}{*}{ ÍTEMS } & \multicolumn{3}{|c|}{ Buenos Durmientes } & \multicolumn{3}{|c|}{ Malos Durmientes } \\
\hline & Mujeres & Varones & Total & Mujeres & Varones & Total \\
\hline Creo que soy buen estudiante & $\begin{array}{c}4,80 \\
(0,95)\end{array}$ & $\begin{array}{c}4,56 \\
(1,15)\end{array}$ & $\begin{array}{l}4,69^{(*)} \\
(1,03)\end{array}$ & $\begin{array}{c}4,23 \\
(1,09)\end{array}$ & $\begin{array}{c}3,82 \\
(1,34)\end{array}$ & $\begin{array}{l}4,10^{(*)} \\
(1,16)\end{array}$ \\
\hline $\begin{array}{l}\text { Disfruto realizando mis tareas } \\
\text { escolares }\end{array}$ & $\begin{array}{c}2,80 \\
(1,44)\end{array}$ & $\begin{array}{c}3,56 \\
(1,59)\end{array}$ & $\begin{array}{c}3,14 \\
(1,53)\end{array}$ & $\begin{array}{c}3,00 \\
(1,47)\end{array}$ & $\begin{array}{c}2,28 \\
(0,95)\end{array}$ & $\begin{array}{c}2,75 \\
(1,33)\end{array}$ \\
\hline Pienso acabar el Bachiller & $\begin{array}{c}5,50 \\
(1,19)\end{array}$ & $\begin{array}{c}5,37 \\
(1,48)\end{array}$ & $\begin{array}{c}5,44 \\
(1,16)\end{array}$ & $\begin{array}{c}5,61 \\
(1,12)\end{array}$ & $\begin{array}{c}5,43 \\
(1,51)\end{array}$ & $\begin{array}{c}5,55 \\
(1,23)\end{array}$ \\
\hline $\begin{array}{l}\text { Estoy interesado en asistir a } \\
\text { la Universidad }\end{array}$ & $\begin{array}{c}5,30 \\
(1,34)\end{array}$ & $\begin{array}{c}5,47 \\
(0,83)\end{array}$ & $\begin{array}{c}5,37 \\
(1,14)\end{array}$ & $\begin{array}{c}5,31 \\
(1,55)\end{array}$ & $\begin{array}{c}5,28 \\
(1,89)\end{array}$ & $\begin{array}{c}5,30 \\
(1,62)\end{array}$ \\
\hline Tengo buenas calificaciones & $\begin{array}{c}4,85 \\
(1,14)\end{array}$ & $\begin{array}{c}4,00 \\
(1,26)\end{array}$ & $\begin{array}{c}4,47 \\
(1,25)\end{array}$ & $\begin{array}{c}4,46 \\
(1,05)\end{array}$ & $\begin{array}{c}4,00 \\
(1,26)\end{array}$ & $\begin{array}{c}4,31 \\
(1,11)\end{array}$ \\
\hline $\begin{array}{l}\text { Tengo problemas con mis } \\
\text { compañeros/as de clase }\end{array}$ & $\begin{array}{c}5,70 \\
(0,73)\end{array}$ & $\begin{array}{c}5,50 \\
(1,03)\end{array}$ & $\begin{array}{l}5,61^{* *} \\
(0,87)\end{array}$ & $\begin{array}{c}5,00 \\
(1,58)\end{array}$ & $\begin{array}{c}5,00 \\
(1,15)\end{array}$ & $\begin{array}{l}5,00^{(*)} \\
(1,41)\end{array}$ \\
\hline $\begin{array}{l}\text { Tengo problemas con los profesores } \\
\text { de la escuela }\end{array}$ & $\begin{array}{c}5,85 \\
(0,37)\end{array}$ & $\begin{array}{c}5,37 \\
(0,88)\end{array}$ & $\begin{array}{c}5,63 \\
(0,68)\end{array}$ & $\begin{array}{c}5,46 \\
(1,13)\end{array}$ & $\begin{array}{c}5,14 \\
(1,86)\end{array}$ & $\begin{array}{c}5,35 \\
(1,39)\end{array}$ \\
\hline $\begin{array}{l}\text { Me siento rechazado/a por mis } \\
\text { compañeros/as de clase }\end{array}$ & $\begin{array}{l}5,85^{(* *)} \\
(0,67)\end{array}$ & $\begin{array}{c}5,68 \\
(0,79)\end{array}$ & $\begin{array}{l}5,78^{(* *)} \\
(0,72)\end{array}$ & $\begin{array}{c}4,84 \\
(1,67)\end{array}$ & $\begin{array}{c}5,43 \\
(1,13)\end{array}$ & $\begin{array}{l}5,05^{(* *)} \\
(1,50)\end{array}$ \\
\hline $\begin{array}{l}\text { Creo que mis compañeros/as } \\
\text { se burlan de mi }\end{array}$ & $\begin{array}{c}5,80 \\
(0,69)\end{array}$ & $\begin{array}{c}5,69 \\
(0,60)\end{array}$ & $\begin{array}{l}5,75 \\
(0,65)\end{array}$ & $\begin{array}{c}5,00 \\
(1,68)\end{array}$ & $\begin{array}{c}6,00 \\
(0,01)\end{array}$ & $\begin{array}{c}5,35 \\
(1,42)\end{array}$ \\
\hline Creo que la escuela es aburrida & $\begin{array}{c}3,60 \\
(1,35)\end{array}$ & $\begin{array}{c}3,87 \\
(1,67)\end{array}$ & $\begin{array}{c}3,72 \\
(1,48)\end{array}$ & $\begin{array}{c}3,65 \\
(1,75)\end{array}$ & $\begin{array}{c}4,22 \\
(1,69)\end{array}$ & $\begin{array}{c}3,48 \\
(1,67)\end{array}$ \\
\hline
\end{tabular}

C5-Perturbaciones del sueño $(\mathrm{r}=-0,280$, $\mathrm{p}=0,036)$ y C7-Disfunción diurna $(\mathrm{r}=-0,381$, $\mathrm{p}=0,004)$. Esta última variable mostró correlación con otras cuatro variables del EBAE10: "Tengo problemas con los profesores" $(\mathrm{r}=-0,309, \mathrm{p}=0,021)$, "Siento rechazo de mis compañeros" $(\mathrm{r}=-0,311, \mathrm{p}=0,02)$, "Disfruto realizando mis tareas" ( $\mathrm{r}=-0,440, \mathrm{p}=0,001)$ y "La escuela es aburrida" ( $\mathrm{r}=-0,274, \mathrm{p}=0,041)$.

\section{DISCUSIÓN}

El presente estudio aborda la interrelación entre calidad, hábitos de sueño, ajuste y rendimiento escolar en un grupo de adolescentes. Lo más relevante es detectar una alta presencia de somnolencia diurna y su relación con algunos hábitos de sueño y variables de ajuste escolar. 
Más de un tercio de los adolescentes son malos durmientes y la disfunción diurna es el componente con peores resultados.

En este estudio, la presencia de problemas del sueño (malos durmientes, según el ICSP) alcanza al $35,7 \%$ de la muestra, cifra no muy distinta a las publicadas en otras cohortes ${ }^{(33,34,35,36)}$.

La disfunción diurna, en términos de somnolencia, es el componente del ICSP que muestra mayor alteración de todo el índice durante el estudio. La somnolencia está más presente en los que refieren mayor dificultad para despertarse. Es percibida por más de la mitad de la muestra como un problema del sueño, recibiendo la mayor cantidad de respuestas desfavorables extremas.

En nuestro estudio, la somnolencia encontrada $(55,4 \%)$ coincide con el 53\% registrado entre los 1.155 participantes de 11 a 18 años de otra investigación española ${ }^{(37)}$, y difiere ligeramente de otros estudios en población no española, con porcentajes entre el $41,9 \%$ y el $45,7 \%{ }^{(38,39)}$. De nuestros resultados destacamos la baja percepción de los adolescentes sobre la severidad de la somnolencia. Sólo el $19,5 \%$ considera la somnolencia un problema realmente importante para el desempeño de sus tareas diarias, siendo su severidad del 0,8 en la escala de $0-3$, y pese a referirla con una frecuencia promedio alta, entre 1 y 2 veces por semana (dato extraído del análisis del componente C7-Disfunción Diurna).

Este porcentaje coincide con el estudio realizado por la National Sleep Foundation en EEUU $^{(40)}$, en el que un $20 \%$ de los adolescentes perciben la somnolencia como un problema, siendo esta percepción mayor en las mujeres y a medida que aumenta la edad ${ }^{(39)}$.

Las mujeres duermen, y creen que deben dormir, menos respecto a los varones. Un porcentaje significativo de ambos sexos se resisten a ir a la cama y tienen dificultad para despertarse, según sus padres.

Uno de los factores señalados como causa de somnolencia es la duración del sueño ${ }^{(18)}$. Expertos de la National Sleep Foundation recomiendan que los adolescentes entre 14 y 17 años duerman entre 8 y 10 horas diarias ${ }^{(41)}$. En nuestro estudio, la duración media del sueño en toda la muestra ( 7 horas y 45 minutos) es ligeramente inferior a dicha recomendación. Comparando por sexo, los varones cumplen mejor que las mujeres la recomendación de la fundación americana.

Ambos sexos rompen la rutina del sueño los fines de semana, retrasando su inicio 1 hora $\mathrm{y}$ 40 minutos, dato similar a lo descrito en alumnos de 11 a 18 años de Cuenca ${ }^{(42)}$. Esto refleja el conocido patrón de sueño irregular de los adolescentes, con un componente de corta duración del sueño en los días escolares y otro de recuperación los fines de semana ${ }^{(43)}$.

Casi la mitad de los adolescentes de la muestra presentan dificultad para despertarse por las mañanas, de los cuales menos de la mitad lo pueden justificar por el hecho de haberse acostado tarde. Esta supuesta relación entre resistencia a dormir y dificultad para despertarse parece más evidente en los varones que en las mujeres. Esto sugiere que otros factores podrían intervenir en la dificultad para despertarse de los adolescentes y una posible diferencia entre los sexos.

El conocido retraso en la secreción de melatonina que retarda el ciclo del sueño en los adolescentes justificaría la tendencia a dormir y despertarse más tarde ${ }^{(44)}$. Sin embargo, la liberación de esta hormona reviste un carácter individual que podría explicar la diferencia entre sujetos. Los patrones del sueño son explicados por la interacción de factores genéticos, 
conductuales, ambientales y sociales ${ }^{(45)}$. En el caso de las mujeres, además, el sueño puede verse afectado por las influencias hormonales durante el síndrome premenstrual, percibiéndose una peor calidad del sueño ${ }^{(46)}$.

El número de horas ideales que los adolescentes piensan que deberían dormir no aparece relacionada con variables como la C7-Disfunción diurna, las "dificultades para despertarse" o la "resistencia a irse a la cama", ni con ninguno de los otros componentes de ICSP, y se correlaciona negativamente con la duración real del sueño. Ello sugiere que la discrepancia entre lo que duermen realmente los adolescentes y lo que ellos creen que deberían dormir está relacionada con su propia percepción de la duración del sueño. Esta percepción es útil para promover mejores hábitos a la hora de dormir.

Los varones tienen menor rendimiento académico, independientemente del ICSP. Malos y buenos durmientes sienten que disponen de buen ajuste escolar, aunque de menor motivación en sus tareas. La calidad subjetiva del sueño, las perturbaciones del sueño y, especialmente, la disfunción diurna se relacionan negativamente con el ajuste escolar.

Si bien no puede demostrarse la relación entre los problemas del sueño y el rendimiento académico de los adolescentes debido, probablemente, al pequeño número de malos durmientes, si se reconocen diferencias entre las mujeres respecto de los varones como grupo con mejores notas. Este resultado coincide con el informe publicado por el Ministerio de Educación, Cultura y Deporte (2012) ${ }^{(47)}$ [actual Ministerio de Cultura y Formación Profesional] y difiere de otros realizados en Galicia y Aragón, donde las mujeres tienen mejores resultados en lengua y los varones en matemáticas ${ }^{(48,49)}$.

De manera general, los estudiantes muestran un buen ajuste escolar, seis variables del
EBAE-10 reflejan promedios por encima de 5 puntos de una puntuación máxima de 6 . Sólo las dos preguntas que hacían referencia a disfrutar con las tareas de clase o de la escuela denotan en general cierta falta de motivación, al obtenerse promedios más bajos, independientemente del valor de ICSP o del sexo.

Los problemas, como el rechazo de los compañeros o el sentirse peor estudiante, si bien son mayores en los malos durmientes de manera estadísticamente significativa, los consideramos poco relevantes en la práctica, porque el grado de ajuste escolar en cualquiera de esas variables es siempre muy alto en buenos y malos durmientes (valores entre 4,10-5,78 de 6 puntos máximos). Esto contrasta con lo sucedido para las variables "disfrutar con las tareas" y "la clase es aburrida", donde los puntos obtenidos son 2,14-3,72 de 6 puntos máximos posibles, como se observa en la tabla 5 .

En todo caso, la asociación entre variables que expresaron ajuste escolar (rechazo-problemas con los compañeros o con los profesores, disfrute de tareas escolares) con las variables que identificaron dificultades del sueño (calidad subjetiva del sueño, perturbaciones del sueño y disfunción diurna) sitúan a la somnolencia, implícita en la disfunción diurna, como un elemento central en estas asociaciones. En este sentido, la disfunción diurna muestra la asociación negativa más alta de todas las correlaciones con la variable "disfruto realizando las tareas escolares" $\mathrm{y}$, además, tiene mayor número de asociaciones significativas con otras variables del ajuste escolar.

La baja disposición, en general, de los adolescentes hacia las tareas y actividades de la escuela puede empeorar debido a la somnolencia y a la peor calidad del sueño ${ }^{(34,50)}$. Incluso, puede afectar al estado anímico y hacer al alumno más irritable ${ }^{(51)}$. La somnolencia parece implicada en diversos aspectos del individuo como la baja 
atención, los cambios emocionales o conductuales y el bajo rendimiento académico ${ }^{(3,52)}$.

En un estudio con 3.235 alumnos canadienses, realizado entre 1998-2001, el 23\% de los alumnos percibieron que sus calificaciones empeoraron a causa de la somnolencia ${ }^{(53)}$. En España, los adolescentes con quejas de sueño suspenden más asignaturas frente a los que no (2,28 asignaturas contra 1,91$)$, así como también los que se levantan cansados $(2,17$ frente a 1,97$) \mathrm{y}$, especialmente, los que tienen somnolencia diurna $(2,17 \text { contra } 1,75)^{(42)}$.

Como limitaciones de nuestro estudio subrayamos el uso de un diseño que no permite establecer relaciones de causalidad y la subjetividad implícita en la valoración por cuestionarios, así como no recoger de forma exhaustiva aspectos relacionados con la higiene del sueño, los cuales deberán tenerse en cuenta para investigaciones futuras, donde factores complejos como la somnolencia requieren profundizar en su estudio.

Como conclusión, podemos afirmar que, en nuestro entorno, la somnolencia es un problema relativamente frecuente y poco valorado por los propios alumnos que puede influir en el ajuste escolar y en su motivación para realizar las actividades escolares. Esto demanda una atención activa por parte de todos los elementos involucrados en la formación de los adolescentes. En este trabajo encontramos algunos hábitos del sueño, como la hora de irse a dormir, de despertarse, la falta de rutina en horas de inicio y la duración del propio sueño, que podrían mejorarse.

\section{AGRADECIMIENTOS}

Nuestro agradecimiento a los padres, a los adolescentes participantes y al Instituto de Enseñanza Secundaria de Monelos (La Coruña) por colaborar en la realización de este estudio. A la Dra. MJ López-Armada por su valiosa ayuda en la revisión y redacción final de este artículo.

\section{BIBLIOGRAFÍA}

1. Beebe DW, Rose D, Amin R. Attention, learning, and arousal of experimentally sleep-restricted adolescents in a simulated classroom. J Adolesc Health. 2010;47(5):523-25.

2. Fallone G, Owens JA, Deane J. Sleepiness in children and adolescents: clinical implications. Sleep Med Rev. 2002;6(4):287-306.

3. Curcio G, Ferrara M, De Gennaro L. Sleep loss, learning capacity and academic perfomance. Sleep Med Rev. 2006;10(5)23-37.

4. Drummond SP, Brown GG. The effects of total sleep deprivation on cerebral responses to cognitive performance. Neurophychopharmacology. 2001;25(Supp15):S68-73.

5. Harrison Y, Horne JA. The impact of sleep deprivation on decision making: A review. J Exp Psychol Appl. 2000;6(3):236-49.

6. Wolfson AR, Carskadon MA. Understanding adolescents' sleep patterns and school performance: a critical appraisal. Sleep Med Rev. 2003;7(6):491-506.

7. Martínez-Martínez M, González-Martínez M. Sueño en el adolescente. Rev Adolescere. 2014;2(2):59-66.

8. Dahl RE, Lewin DS. Pathways to adolescent health: Sleep regulation and behavior. J Adolesc Health. 2002;31(Supp16):175-84.

9. Carskadon MA. (2011). Sleep in adolescents: the perfect storm. Pediatr Clin North Am. 2011;58(3):637-47.

10. Liu XC, Zhou H. Sleep duration, insomnia and behavioral problems among Chinese adolescents. Psychiatry Res. 2002;111(1):75-85. 
11. Hysing M, Pallesen S, Sotrmak KM, Lundervold AJ, Siversten B. Sleep patterns and insomnia among adolescents: a population-based study. J Sleep Res. 2013;22(5):549-56.

12. Dewald JF, Meijer AM, Oort FJ, Kerkhof GA, Bögels SM. The influence of sleep quality, sleep duration and sleepiness on school performance in children and adolescents: A meta-analytic review. Sleep Med Rev. 2010;14(3):179-89.

13. Russo PM, Bruni O, Lucidi F, Ferri R, Violani C. Sleep habits and circadian preference in Italian children and adolescents. J Sleep Res. 2007;16(2):163-69.

14.Wolfson AR, Carskadon MA. Sleep schedules and daytime functioning in adolescents. Child Dev. 1998;69(4):875-87.

15.Strauch I, Meier B. Sleep need in adolescents: A longitudinal approach. Sleep. 1988;11(4):378-86.

16. Khalsa SB, Jewett ME, Cajochen C, Czeisler CA. A phase response curve to single bright light pulses in human subjects. J Physiol. 2003;549(3 Pt):945-52.

17. Pin-Arboledas G. Alteraciones del sueño en el niño: enfoque desde la Atención Primaria. Rev Neurol. 2000;30(2):178-86

18. Ohayon MM, Smolensky MH, Roth T. Consequences of shift working on sleep duration, sleepiness and sleep attacks. Chronobiol Int. 2010; 27(3):575-89.

19. Otero-López JM. Consumo de drogas y comportamientos delictivos en la adolescencia. En: Saldaña García C, coord. Detección y prevención en el aula de los problemas del adolescente. Madrid: Pirámide; 2001.p. 179-212.

20. Giorelli AS, Passos dos Santos P, Carnaval Th, Mota Gomes M. Sonolencia excesiva diurnal: aspectos clínicos, diagnósticos y terapéuticos. Rev Bras Neurol. 2012;48(3):17-24

21. Pérez-Lloret S, Videla AJ, Richaudeau A, Vigo, D, Rossi M, Cardinali DP et al. A multi-step pathway connecting short sleep duration to daytime somnolence, reduced attention, and poor academic performance: an exploratory cross-sectional study in teenagers. J Clin Sleep Med. 2013;9(5):469-73.

22. Domínguez F, Soler S, Morell M, Gómez F, Rubio P, Benetó A. Hábitos de sueño en una muestra de población juvenil de la Comunidad Valenciana. Vigilia-Sueño. 2000;12:83-4.

23. Pereira E, Teixeira CS, Louzada FM. Sonolencia diurna excessiva em adolescentes: prevalencia e fatores asociados. Rev Paul Pediatr. 2010;28(1):98-103.

24. Kirmil-Gray K, Eagleston JR, Gibson E, Thorensen CE. Sleep disturbance in adolescents: Sleep quality, sleep habits, beliefs about sleep, and daytime functioning. J Youth Adolesc. 1984;13(5):375-84.

25. Moore M, Lester Kirchner H, Drotar D, Johnson N, Rosen C, Ancoli-Israel S et al. Relationships among sleepiness, sleep time, and psychological functioning in adolescents. J Pediatr Psychol. 2009;34(10):1175-83.

26. Roberts RE, Roberts CR, Duong HT. Sleepness in adolescence: prospective data on sleep deprivation, health and functioning. J Adolesc. 2009;32(5):1045-57.

27. Kiefer S, Ryan A. Striving for social dominance over peers: The implications for academic adjustment during early adolescence. J Educ Psychol. 2008;100(2):417-28.

28. Moral de la Rubia JC, Sánchez Sosa JC, Villarreal González ME. Desarrollo de una escala multidimensional breve de ajuste escolar. REMA. 2010;15(1):1-11.

29. Cabrera L. Revisión sistemática de la producción española sobre rendimiento académico entre 1980 y 2011. RCDE. 2016;27(1):119-39.

30. Concellerías de Participación e Innovación Democrática e Rexeneración Urbana e Dereito á Vivenda do Concello da Coruña. Informe sobre a proposta inicial de reorganización do municipio da Coruña en distritos. A Coruña: Ergosfera. 2016 . Disponible en: http://www.coruna.gal/participacion/ es/procesos-participativos/organizacion-en-distritos. 
31. Buysse DJ, Reynolds CF, Monk TH, Berman SR, Kupfer DJ (1989). The Pittsburgh Sleep Quality Index: a new instrument for psychiatric practice and research. Psychiatric Res. 1989;28(2):193-213.

32. Macías JA, Royuela A. La versión española del Índice de Calidad de Sueño de Pittsburgh. Informaciones Psiquiátricas ICMJE . 1996;146:465-72.

33. Ahrberg K, Dresler M, Niedermaier A, Steiger A, Genzel L. The interaction between sleep quality and academic performance. Psychiatric Res. 2012;46(12):1618-1622.

34. Quevedo-Blasco VJ, Quevedo-Blasco R. Influencia del grado de somnolencia, cantidad y calidad de sueño sobre el rendimiento académico en adolescentes. Int J Clin Health Pychol. 2011;11(1):49-65.

35. Sing CY, Wong WS. Prevalence of insomnia and its psychosocial correlates among college students in Hong Kong. J Am Coll Health. 2010;59(3):174-182.

36. Lund HG, Reider BD, Whiting AB, Prichard JR. Sleep patterns and predictors of disturbed sleep in a large population of college Students. J Adolesc Health. 2010;46(2):124-132.

37. García-Jiménez MA, Salcedo-Aguilar F, RodríguezAlmonacid F M, Redondo-Martínez MP, Monterde-Aznar ML, Marcos-Navarro AI et al. Prevalencia de los trastornos del sueño en adolescentes de Cuenca, España. Rev Neurol. 2004;39(1):18-24.

38. Chung KF; Cheung MM. Sleep-wake patterns and sleep disturbance among Hong Kong Chinese adolescents. Sleep. 2008;31(2):185-194.

39. Pagel JF, Forister N, Kwiatkowki C. Adolescent sleep disturbance and school performance: the confounding variable of socioeconomics. J Clin Sleep Med. 2007;3(1):19-23.

40. National Sleep Foundation. Sleep in America poll. Washington, DC: WBA Market Research; 2006.
41. Hirshkowitz M, Whiton K. Albert SM, Alessi C, Bruni O, DonCarlos L et al. (2015). National Sleep Foundation's updated sleep duration recommendations: final report. Sleep Health. 2015;1(4):233-243.

42. Salcedo Aguilar F, Rodríguez Almonacid FM, Monterde Aznar ML, García Jiménez MA, Redondo Martínez P, Marcos Navarro AI. Hábitos de sueño y problemas relacionados con el sueño en adolescents: relación con el rendimiento escolar. Aten Primaria. 2005;35(8):408-14.

43. Gradisar M, Gardner G, Dohnt, H. Recent worldwide sleep patterns and problems during adolescence: review and meta-analysis of age, region and sleep. Sleep Med. 2011;12(2),110-8.

44. Pin Arboledas G, Morell Safort M, Mompó Marabotto, L. El insomnio del adolescente. En Trastorno del sueño durante la adolescencia. Rev Adolescere. 2013;1(3):30-41.

45. Chaput J. Dutill C, Sampasa-Kanyinga H. Sleeping hours: what is the ideal number and how does age impact this? Nat Sci Sleep. 2018;10:421-430.

46. Baker FC, Kahan TL, Trinder J, Colrain IM. Sleep quality and the sleep electroencephalogram in women with severe premenstrual syndrome. Sleep. 2007;30 (10):1283-91.

47. Ministerio de Educación, Cultura y Deporte. Datos y cifras curso escolar 2010/2011. Madrid: Ministerio de Educación, Cultura y Deporte (MECD); 2012.

48. Gobierno de Aragón. Evaluación del Diagnóstico de Aragón 2011-2012. Informe de resultados de Evaluación Educativa. Zaragoza: Departamento de Educación, Cultura y Deporte; 2012.

49. Xunta de Galicia. Avaliación de Diagnóstico de Galicia 2011-2012. Informe de resultados. Santiago de Compostela: Consellería de Cultura, Educación e Ordenación Universitaria; 2012.

50. Meijer AM, Habekothé HT, Van der Wittenboer GLH. Time in bed, quality of sleep and school functioning of children. J Sleep Res. 2000;9(2):145-53. 
51. Dahl RE. The regulation of sleep and arousal: development and psychopathology. Dev Psychopathol. 1996;8: 3-27.

52. Mitru G, Millrood DL, Mateika JH. The impact of sleep on learning and behavior in adolescents. T Col Rec. 2002;104(4):704-726.

53. Gibson ES, Powles AC, Thabane L, O'Brien S, Molnar DS, Trajanovic N et al. "Sleepiness" is serious in adolescence: two surveys of 3235 Canadian students. BMC Public Health. 2006;6:116. 